\title{
Internet de las Cosas y Visión Artificial, Funcionamiento y Aplicaciones: Revisión de Literatura
}

\author{
(Internet of Things and Artificial Vision, Performance and \\ Applications: Literature Review)
}

\author{
Vanessa Alvear-Puertas ${ }^{1}$, Paul Rosero-Montalvo², Diego Peluffo-Ordóñez ${ }^{3}$, José Pijal-Rojas ${ }^{4}$
}

\begin{abstract}
Resumen:
Internet de las cosas (o también conocido como loT) es una de las tecnologías más nombradas en la actualidad debido a la capacidad que prevé para conectar todo tipo de dispositivos al Internet, si a las potencialidades de loT le adicionamos otra tecnología de alto impacto como lo es la Visión Artificial tenemos un amplio campo de aplicaciones innovadoras, donde el procesamiento de imágenes y video en tiempo real permiten la visualización de grandes cantidades de datos en internet. Las principales aplicaciones que se desarrollan con loT y Visión Artificial pueden ser implementadas en educación, medicina, edificios inteligentes, sistemas de vigilancia de personas y vehículos, entre otros. Este tipo de aplicaciones mejoran la calidad de vida de los usuarios, sin embargo, para su desarrollo se requiere una infraestructura que permita la convergencia de diferentes protocolos y dispositivos, pero de manera especial que puedan manejar las diferentes fases de la adquisición de imágenes. En este trabajo se ha realizado una revisión de los inicios, conceptos, tecnologías y aplicaciones ligados a la Visión Artificial con el Internet de las Cosas para poder comprender de forma precisa el impacto de sus aplicaciones en la vida cotidiana.
\end{abstract}

Palabras clave: IoT; Aplicaciones de loT; Visión Artificial

\begin{abstract}
:
Internet of Things (or also known as loT) is one of the technologies most named today because of the ability it envisages to connect all kinds of devices to the Internet. If to the potentialities of IoT we add another technology of high impact as It is the Artificial Vision we have a wide field of innovative applications, where the processing of images and video in real time allow the visualization of large amounts of data on the Internet. The main applications developed with loT and Artificial Vision can be implemented in education, medicine, intelligent buildings, surveillance systems of people and vehicles, among others. This type of applications improves the quality of life of users, however, for their development an infrastructure is required that allows the convergence of different protocols and devices, but in a special way that can handle the different phases of the acquisition of images. In this work, a review of the beginnings, concepts, technologies and applications related to the Artificial Vision with the Internet of Things has been carried out to be able to understand in a precise way the impact of its application in daily life.
\end{abstract}

Keywords: IOT: IoT applications: artificial vision

\footnotetext{
1 Universidad Técnica del Norte, Ibarra - Ecuador (vealvearp@utn.edu.ec)

2 Universidad Técnica del Norte, Ibarra - Ecuador (pdrosero@utn.edu.ec)

3 Universidad Técnica del Norte, Ibarra - Ecuador (diegohpo@gmail.com)

${ }^{4}$ Instituto Tecnológico Superior 17 de Julio, Urcuquí-Ecuador (jpijal@istyachay.edu.ec)
} 


\section{Introducción}

Internet de las Cosas es considerado como una verdadera revolución en el mundo tecnológico, especialmente en las comunicaciones, según (Chen, Xu, Liu, Hu, \& Wang, 2014) se trata de una red inteligente que permite el intercambio de información y comunicación entre dispositivos inteligentes, por otra parte (Ansari, A. N., Sedky, M., Sedky, M., \& Tyagi, A., 2015) define a loT como una potente plataforma capaz de conectar dispositivos para monitorear aspectos de la vida cotidiana.

En la actualidad se aplica Internet de las Cosas en áreas de salud, construcciones, tráfico vehicular, agricultura, educación, visión artificial, conservación del ambiente, meteorología, etc. Con la fusión de las diferentes áreas de desarrollo de loT se busca fortalecer la formación de Smart Cities, según (Zanella, Bui, Castellani, Vangelista, \& Zorzi, 2014) el Urban loT puede mejorar un sinnúmero de servicios públicos y privados dando como resultado un aprovechamiento de los recursos de una forma eficiente y equilibrada. El término Urban loT se entiende como la tecnología de Internet de las Cosas aplicado a las actividades cotidianas de los ciudadanos y su interacción con los servicios y recursos propios de cada ciudad.

El objetivo de este trabajo es analizar los principales aspectos relacionados a Internet de las Cosas, de manera especial los referentes a Visión Artificial, y las aplicaciones con mayor impacto de loT en la actualidad.

La estructura del presente documento es la siguiente, en la sección 2 se realiza una revisión sobre los conceptos fundamentales de Internet de las Cosas, más adelante en la sección 3 se explican los principios de Visión Artificial y el tratamiento de imágenes; la sección 4 presenta las principales aplicaciones relacionadas a loT y Visión Artificial, dando al lector un enfoque amplio de lo que se está desarrollando gracias a estas tecnologías; a continuación en la sección 5 se plantea la discusión generada a partir de este trabajo y finalmente se presentan las conclusiones en la sección 6.

\section{Internet de las Cosas (IoT)}

Con el transcurso del tiempo el concepto de loT se ha ido cambiando y transformando. En el 2005, la Unión Internacional de Telecomunicaciones (UIT) publicó su primer informe sobre el loT (ITU, 2005). Donde menciona al Internet de los Cosas como "Una promesa de un mundo de dispositivos interconectados que proveen contenido relevante a los usuarios" de esta manera se da a conocer al mundo el nuevo término. 
En julio de 2012, la UIT declaró un nuevo concepto de IoT (ITU, 2012) "Infraestructura global para la sociedad de la información, que permite que los servicios de interconexión (física y virtual), cosas u objetos existentes y en evolución coexista en un medio".

Cisco en el 2013 define al loT como la interacción entre los seres humanos y los objetos en diversas aplicaciones, tales como la fabricación, la logística, el sector de los servicios, la agricultura, el reciclaje, gestión ambiental, casas inteligentes y otros, añadiendo una nueva forma de conectividad de los "objetos" a Internet (CISCO, 2013).

Internet de las Cosas (IOT) ha evolucionado con la finalidad de unir a un gran número de elementos con diferentes capacidades de conexión y la computación a una gran y única red, siendo estos capaces de enviar información sobre la Internet, detectar el estado de un entorno, procesar los datos y enviar los resultados.

a. Dificultades de loT: Los dispositivos "objetos" se expanden cada día, dando lugar para que se extienda a todas las industrias y servicios ocasionando inconvenientes que se citan a continuación:

- La seguridad y privacidad. -Temas críticos ya que cada objeto puede ser un punto de infección o vulnerabilidad para la toda la red (B. Fabian and O. Gunther, 2007).

- Movilidad.- El loT deberá proporcionar una conectividad sin fisuras, independientemente de donde los objetos se colocan o se trasladen, además de tener en cuenta la importancia de no perder datos por ser un objeto móvil (I. F. Akyildiz, 2007).

- Limitación de recursos: Es necesario mencionar que cada objeto necesita gestionar su propia energía y capacidad de almacenamiento (D. Guinard, 2010) .

- La heterogeneidad: Es una de las principales dificultades ya que se necesita que todos los objetos conectados a la red hablen el mismo idioma, es decir que existan protocolos para que converjan todos los dispositivos y puedan comunicarse dentro de la red loT (Michele Nitti, 2015).

- Plug and Play: Tan pronto como un dispositivo se une a una red, debe registrarse y entrar en funcionamiento permitiendo que todos los objetos puedan comunicarse con el sin ningún inconveniente. En sistemas de 10 (Entradas/Salidas), el desafío es hacer este proceso automático y dinámico que permita al objeto interactuar inmediatamente con otros objetos sin la necesidad de intervención humana (Michele Nitti, 2015).

b. Arquitecturas de IOT: Uno de los principales problemas en la IO es que su concepto es muy amplio de tal manera que no se propone ninguna arquitectura uniforme (Somayya Madakam, 2015). (Bagula, 2013) y (Department of Electrical and Electronic Engineering - University of Cagliari, 2015) plantean las siguientes arquitecturas: 
- Arquitectura UIT. -De acuerdo con las recomendaciones de la Unión Internacional de Telecomunicaciones (UIT), la arquitectura de loT consiste en (Bagula, 2013): Capa de Acceso, Capa de Red, Capa Inter-Lógica y Capa Aplicación

- Arquitectura de 3 capas. - La arquitectura loT hace uso de la representación de un modelamiento en capas y se divide básicamente en 3 con funciones independiente (Department of Electrical and Electronic Engineering - University of Cagliari, 2015): Capa Física, Capa de Virtualización y Capa de Aplicación.

c. Protocolos de loT: IoT en su capa de aplicación implementa dos nuevos protocolos MQTT y CoAP. El protocolo MQTT (este nombre proviene de MQ Telemetry Transport) es una implementación realmente ligera y sencilla de un protocolo de mensajería, su diseño se orienta a redes con bajo ancho de banda y altos niveles de latencia. Por otra parte, el protocolo de aplicación restringida (COAP) es un protocolo de transferencia especializado para el uso con nodos y redes restringidas, entiéndase por restringidas aquellas que presentan baja potencia y altos índices de pérdidas. CoAP está diseñado para interactuar fácilmente con HTTP para la integración con la Web y su operación se basa en el protocolo UDP de capa transporte del modelo TCP/IP (Jaffey, 2014).

\section{Visión Artificial}

La visión artificial o como la llaman otros autores visión por computador, se la define como la ciencia de programar un computador para procesar imágenes o videos e incluso entenderlos (Culjak \& Abram, 2012). En (Bradski \& Kaebler, 2008) explica que es la transformación de datos desde un fotograma o video cámara en lo que puede ser una decisión o una nueva representación. En la Figura 1 se presenta paso a paso el proceso que se ejecuta en la visión artificial, indicando el orden de cada fase que va desde la adquisición de la imagen y el procesamiento hasta la ejecución de una acción en particular con los datos obtenidos. 


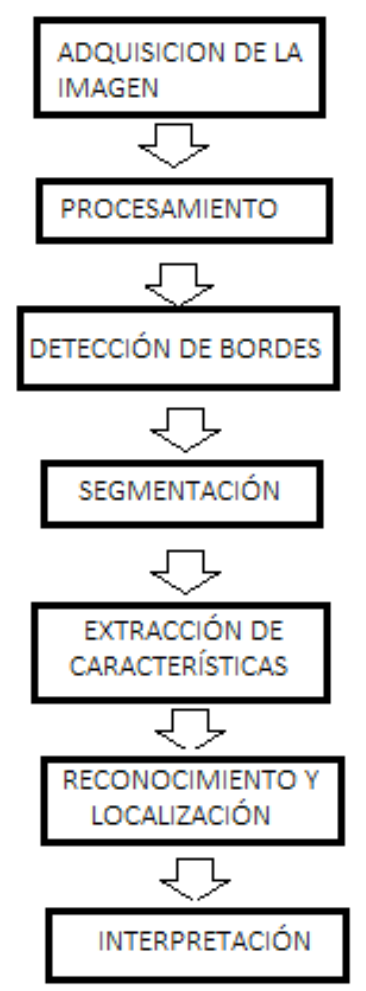

Figura 1. Fases del proceso de funcionamiento de la visión artificial al determinar una imagen

Dado que los usos de estas fases no dependen una de otra ni tampoco son obligatorias, influyen en el trabajo que se realice, explica (Álvarez, 2014).

En la fase de adquisición de la imagen, se indica en (Vélez, Moreno, Sánchez, \& Sánchez, 2003) que es la captura y digitalización de la imagen de lo físico a lo digital. Una vez que la imagen se ha digitalizado, esta entra a un pre procesamiento el cual facilita etapas posteriores, eliminando partes de la imagen que no se toman en cuenta según (Álvarez, 2014). Una de las etapas importantes en este proceso es la detección de bordes el cual discrimina el fondo, separando los objetos de interés reduciendo así los datos indica (Shah, 1997). A continuación, se realiza una segmentación de la imagen, donde (Posada, 2015) indica que se puede seleccionar los pixeles en función de sus valores sean RGB o HSV. RGB, por sus siglas en inglés Red, Green, Blue, es un modelo de color que permite representar todos los colores basándose en los colores primarios de la luz. HSV por sus siglas en inglés Hue, Saturation, Value, es un modelo de color que se basa en componentes que son matiz, saturación y valor para combinar distintos colores. De esta manera el autor indica que es posible destacar un objeto de un determinado color. Por último (Álvarez, 2014) indica que el reconocimiento y localización es donde se clasifican los objetos con características comunes dentro del espacio 3D y restringir el espacio mediante técnicas de triangulación para que se seleccione una única imagen y esta pueda ser interpretada. 
a. Procesamiento de la imagen: Dentro de la visión artificial es necesario un tratamiento de la imagen con la finalidad de facilitar procesos posteriores. Los propósitos a continuación explicados por (Cárdenas \& Lerena, 2012) son:

- Disminuir el ruido producido por los dispositivos electrónicos.

- Mejorar la calidad de algunas partes de la imagen.

- Resaltar las características de la imagen.

- Transformar la imagen a otro espacio de representación.

b. Smoothing: Es el suavizado de la imagen, es muy utilizado en procesamiento de imagen para la reducción de ruido o ayudar a identificar los bordes de la imagen (Matuska, Hudec, \& Miroslav, 2012).

c. Conversión a Escala de Grises: En el trabajo de (Sobrado, 2009) indica que la conversión de una imagen a escala de grises es el equivalente a la luminancia de la imagen. En este proceso es importante la intensidad de la luz para poder identificar los colores del espectro visible para el ojo humano. Mientras que (Cárdenas \& Lerena, 2012) enfatiza que la conversión a escala de grises se realiza a través del uso de una media ponderada de los componentes de color de cada pixel. En la Figura 2 se presenta un ejemplo de conversión a escala de grises previo al tratamiento de la imagen.

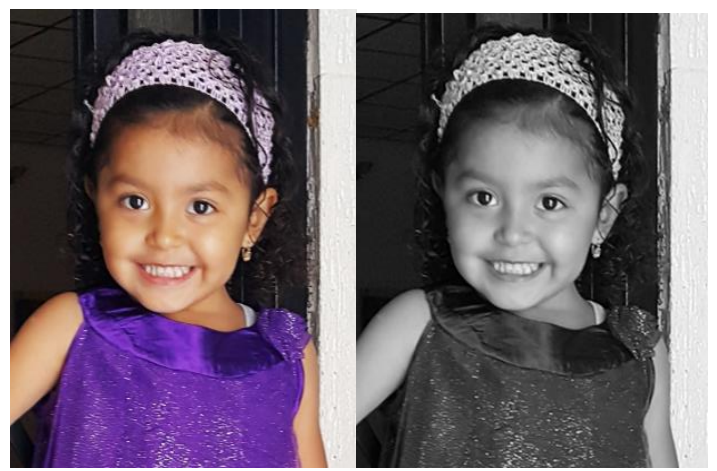

Figura 2. Imagen a colores e imagen en escala de grises

d. Morfología de la Imagen: Las transformaciones morfológicas básicas se llaman dilatación y la erosión, y se presentan en una amplia variedad de contextos, tales como la eliminación de ruido, el aislamiento de los elementos individuales, y elementos de unión diferentes en una imagen. La morfología también se puede utilizar para encontrar protuberancias o agujeros de intensidad en una imagen y para encontrar gradientes de imagen. Dilatación es una convolución de algunas imágenes (o región de una imagen) La erosión es la operación inversa la dilatación, esto lo indica (Bradski \& Kaebler, 2008). En la Figura 3 se puede observar una imagen a colores con su respectiva morfología. 


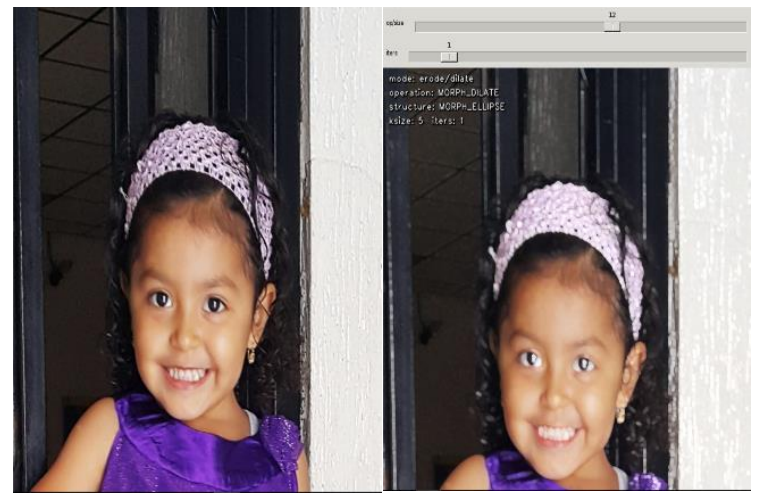

Figura 3. Imagen a colores y morfología de la imagen

e. Detección de Bordes: La detección de bordes es una importante etapa de pre-procesamiento de análisis de imágenes indica (Matuska, Hudec, \& Miroslav, 2012). Inicialmente es necesario encontrar en toda la imagenla región de interés, para esto se implementa un algoritmo de detección de contornos, que encuentra los bordes de la imagen, buscando los cambios entre tonalidades, en este caso para la imagen binarizada son los bordes del cambio de color entre el blanco y el negro (Bradski \& Kaebler, 2008) . Esto nos permite acceder a cada contorno no solo para visualizarlo sino para filtrarlo con diferentes criterios de clasificación según los atributos (Posada, 2015).

\subsection{Detección facial con Visión Artificial}

Los sistemas de detección facial han tomado fuerza en los últimos años debido a que son ampliamente utilizados en sistemas biométricos, el objetivo de estos sistemas es identificar a un individuo en una imagen digital de forma automática. La detección facial automática tuvo sus inicios en los años 60, el primer sistema semiautomático necesitaba que el administrador localice rasgos como ojos, cejas, nariz y boca en las imágenes para calcular distancias a determinados puntos de referencia que después serían analizados con datos almacenados en el sistema. Los próximos sistemas de detección usaron distintos marcadores y técnicas para mejorar el proceso de detección. En la actualidad existen sofisticadas herramientas que son aplicadas en distintas áreas sociales como entretenimiento, tarjetas inteligentes (pasaportes, licencias de conducir), seguridad de la información, aplicación de la ley y vigilancia, entre otros.

A pesar de que la detección facial es una tarea cotidiana para el ser humano, la implementación de un software no ha sido tarea fácil y ha requerido amplias investigaciones que mejoren el proceso. El desarrollo e implementación de los algoritmos requeridos para los sistemas de detección facial se han subdividido en áreas específicas: detección de rostro, seguimiento, alineamiento, extracción de rasgos, entrenamiento, identificación, clasificación, análisis de expresión facial, análisis en 2 y 3 dimensiones (García, 2009). 


\section{Aplicaciones de loT y Visión Artificial}

El campo de aplicaciones de loT se ha extendido por diversas áreas de estudio, permitiendo usar esta tecnología para la mejora de recursos naturales y tecnológicos e incluso mejorar ciertas actividades de la vida cotidiana. A continuación, se citan algunos trabajos que vinculan loT de la mano de la visión artificial.

a. Conteo de personas en edificios inteligentes: El trabajo realizado por (Paci , Brunelli, \& Benini, 2014) nace de la necesidad de implementar un sistema de conteo de personas dentro de un edificio, aunque aún esta tecnología no ha tenido muchos casos de estudio, se presenta como un requisito para mejorar las condiciones medio ambientales y de seguridad dentro de lugares donde se tiene un alto número de ocupantes. Para los autores de este trabajo, su caso de estudio específico se enfoca dentro de las aulas de clases, donde buscan brindar un mayor bienestar a los estudiantes mientras permanecen en sus aulas, y a la vez generar un ahorro de energía, para esto han implementado dos tipos de nodos, el primer nodo es el de sensores y el segundo es el de las cámaras. El funcionamiento de este sistema se basa en un algoritmo para conteo de personas que se ejecuta mediante el uso de cámaras y visión artificial, además el sistema en su nodo de sensores monitorea condiciones ambientales como temperatura y humedad, todos los datos son transmitidos inalámbricamente a través de una Red Inalámbrica de Sensores mejor conocida como WSN. Los modelos de ocupación al ser combinados con los datos ambientales permiten un control automatizado de los sistemas de aire acondicionado generando a la par un ahorro de energía. Por otra parte, el conteo de personas mejora la seguridad dentro de estos edificios.

Estudios similares fueron desarrollados por (Akkaya, Guvenc, Aygun, \& Pala, 2015), en este caso se consideraron como punto de enfoque el creciente número de edificios inteligentes.

b. Parqueo inteligente y reducción de tráfico vehicular: Uno de los pilares del concepto Smart City es el manejo inteligente del tráfico vehicular, al respecto se han realizado numerosos trabajos tal como el realizado por (Baroffio, Bondi, Cesana, Redondi , \& Tagliasacchi, 2015) la solución propuesta en este trabajo se centra en el uso de WSNs y una adaptación a esta tecnología empleando visón artificial que da como resultado una VSN (Visual Sensor Network). Un nodo VSN es aquel que está conectado a una cámara, por lo cual puede adquirir, procesar y capturar imágenes y videos de un lugar específico y trasmitir esa información a su nodo central. Esta tecnología es empleada para implementar un sistema de información para estacionamiento que localice los espacios disponibles y los difunda entre los conductores reduciendo significativamente el tráfico dentro de las zonas 
congestionadas. El sistema está compuesto por una red inalámbrica de nodos con cámaras que ejecutan un algoritmo de detección de espacios disponibles, el procesamiento de las imágenes se realiza en el nodo central y desde aquí se transmite la información a los conductores, esta es una solución en tiempo real que vincula el concepto de visión artificial e loT en beneficio de la población.

c. Sistemas de vigilancia y control en hogares: loT requiere tan solo de una cámara y ciertos algoritmos para poder realizar el análisis de imágenes y videos, si bien es cierto las cámaras pueden ser de distintas características, pero lo que se requiere específicamente es capturar imágenes. Basados en esta idea (Ansari, A. N., Sedky, M., Sedky, M., \& Tyagi, A., 2015) han implementado un sistema de vigilancia para domicilios usando una placa Raspberry Pi y una cámara web, con un costo de aproximadamente 60 dólares se pueden obtener imágenes en tiempo real, el sistema cuenta con un proceso de notificación mediante correo electrónico o mensajería que le indicará al propietario si dentro de su hogar se ha detectado un movimiento. El sistema se basa en el uso de scripts en Python y un almacenamiento de información en un servidor FTP externo. Esta solución es una alternativa sencilla y económica pero que brinda resultados eficientes como sistema de seguridad y se adapta al concepto de Smart Home.

d. Medicina: En el campo de la medicina mediante la visión artificial se puede clasificar o visualizar una imagen de mejor forma, con imágenes procesadas en alta resolución se puede operar desde lugares remotos sin tener la necesidad de que un doctor especializado deba trasladarse al lugar de la cirugía.

e. Inspección de Calidad: La inspección puede involucrar los sectores de la verificación de dimensiones y fallas de un elemento, las cámaras monitoreando la producción en serie de una fábrica, el control se lo realiza fuera o dentro de las instalaciones, este proceso puede ser revisado mediante cualquier dispositivo móvil con acceso a las direcciones públicas de la empresa, con el internet de las cosas se pueden activar o desactivar máquinas dando un mejor un control de la producción.

f. IoT con Visión Artificial en la Educación: Una alternativa para mejorar la educación desde los niveles iniciales hasta las aulas universitarias es involucrar a los estudiantes con las TICs, en el caso específico de loT se pueden desarrollar herramientas didácticas de aprendizaje que cuenten con una plataforma en internet donde los resultados alcanzados por el estudiante puedan ser visualizados en tiempo real. El uso de visión artificial es una herramienta que podría implementarse para monitorear a los alumnos, determinando estados de ánimo, cansancio e incluso desconcentración que se pueden conocer al evaluar y reconocer ciertos 
patrones fisiológicos de comportamiento Los beneficios de este tipo de monitoreo permiten determinar mediante modelos predictivos y algoritmos de Machine Learning (Aprendizaje de Máquina) cuáles son los periodos de tiempo y los horarios más adecuados para alcanzar un aprendizaje óptimo.

Dentro de este campo de investigación se desarrolló un sistema de monitoreo facial que brinda estimadores de desconcentración del estudiante universitario dentro del aula de clase, con la implementación de scripts de Python y empleando la herramienta OpenCV, se desarrolló un sistema capaz de determinar el número de bostezos y número de parpadeos de los estudiantes, debido a que estas variables son indicadores de cansancio y por ende se relacionan a la desconcentración, posterior a un análisis de datos se determinó si el estudiante está desconcentrado o no; estos datos son subidos a una plataforma en Internet, donde los docentes pueden conocer cómo interactúan sus alumnos durante los periodos académicos. Una vez realizada la fase de pruebas se determinó los horarios adecuados para que los estudiantes aprovechen de mejor manera sus procesos de enseñanza, mejorando el proceso de aprendizaje con el uso de estas herramientas tecnológicas (Alvear, 2016). En la Figura 4 se presenta una prueba del monitoreo facial realizada con los estudiantes dentro del proceso de validación del sistema.

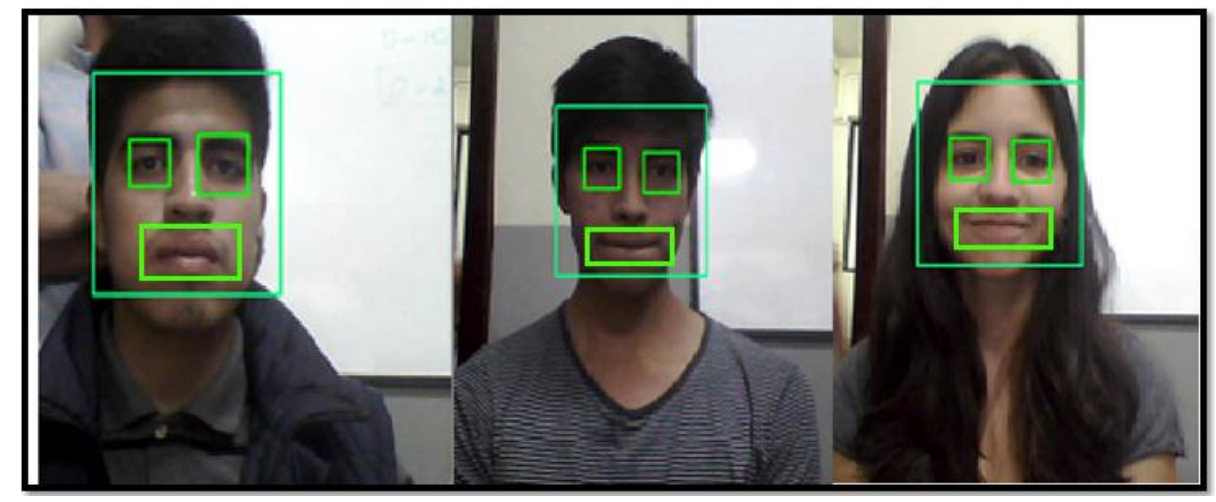

Figura 4. Prueba realizada con el sistema de monitoreo facial que emplea loT y Visión Artificial

\section{Discusión}

El loT pretende que los datos que se obtengan por medio de sensores puedan ser monitoreados de forma remota, de esta manera generar bases de datos con las cuales se pueda realizar estadísticas, tendencias, probabilidades, todo esto en función de comportamientos y actividades de personas o ambientes donde se puede aplicar sistemas electrónicos completos y obtener estos datos en busca de mejorar procesos o determinar puntos débiles.

El campo de la visión artificial en la actualidad va de la mano con la inteligencia artificial donde ya no solo se determinan ciertos parámetros de una imagen, sino que una computadora sea capaz de describir una imagen. Todo parte desde la base del procesamiento de imágenes que es 
indispensable, de tal manera que la interpretación de una imagen o la captura de videos puedan ser procesados para mejor desempeño de un algoritmo.

\section{Conclusiones}

Con la revisión del estado del arte de loT y Visión Artificial se han evidenciado que los estudios y avances tecnológicos en un futuro no muy lejano serán implementados a la vida cotidiana de las personas y se podrá convivir con ello. Estas tecnologías buscan brindar confort y dar facilidades en los diferentes ambientes en que residimos.

Con el abundante cambio e incremento de la tecnología con la finalidad de mejorar y facilitar la vida diaria del ser humano el concepto de loT cada vez está más presente. La necesidad de siempre estar conectado a nuestros objetos permitirá innumerables aplicaciones de loT en todos los dominios y campos de estudio que incluyen la medicina, industria, educación, vivienda, minería, hábitat, transporte, etc. Pero la aplicación de la loT se ve limitada al no tener una arquitectura y estándares para que todas puedan interactuar entre sí, además el internet de las cosas requiere grandes esfuerzos para abordar y presentar soluciones para su seguridad y amenazas a la privacidad.

Los dispositivos electrónicos CCTV (Circuito Cerrado de Televisión) en los hogares e incluso cámaras de seguridad en las ciudades podrán utilizar la visión artificial para dejar de ser dispositivos limitados a grabar videos únicamente, al contrario, se contará con ayuda para detectar accidentes como enviar una alerta si un niño cae en una piscina y no simplemente grabarlo como seria lo común. Determinar en las calles accidentes automovilísticos y enviar alerta a las ambulancias y que este les informe de la situación y no esperar que alguien llame vía telefónica.

\section{Bibliografía}

Akkaya, K., Guvenc, I., Aygun, R., \& Pala , N. (2015). loT-based occupancy monitoring techniques for energy-efficient smart buildings. Wireless Communications and Networking Conference Workshops (WCNCW), 2015 IEEE, (págs. 58-63). New Orleans, LA.

Álvarez, M. (2014). Análisis, diseño e implementación de un sistema de control de ingreso de vehículos basado en visión artificial. Cuenca.

Alvear, V. (2016). Sistema de Monitoreo Facial que brinde estimadores de desconcentración del estudiante universitario dentro del aula de clase a escala de laboratorio. 
Ansari, A. N., Sedky, M., Sedky, M., \& Tyagi, A. (2015). An Internet of things approach for motion detection using Raspberry Pi. Proceedings of 2015 International Conference on Intelligent Computing and Internet of Things, ICIT 2015, (págs. 131-134).

B. Fabian and O. Gunther. (2007). "Distributed ons and its impact on privacy,. IEEE International Conference, 1223-1228.

Bagula, D. A. (2013). INTERNET OF THE THINGS (IOT):An introduction to wireless sensor. ISAT Laboratory, University of Cape Town, South Africa , 43. Obtenido de http://wireless.ictp.it/school_2013/Lectures/Internet-of-the-Things-Middleware-IntroductionICTP-March2013-latest.pdf

Baroffio, L., Bondi, L., Cesana, M., Redondi , A., \& Tagliasacchi, M. (2015). A Visual Sensor Network for Parking Lot Occupancy Detection in Smart Cities. Internet of Things (WF-IOT), 2015 IEEE 2nd World Forum on, (págs. 745 - 750). Milan.

Bradski, G., \& Kaebler, A. (2008). Learning OpenCV. Cambridge: O’Reilly Media, Inc.

Cárdenas, M., \& Lerena, O. (2012). Automatizacion de un sistema de centrado de componentes utilizando visión artificial. Cuenca: UPS.

Chen, S., Xu, H., Liu, D., Hu, B., \& Wang, H. (2014). A Vision of loT: Applications, Challenges, and Opportunities With China Perspective. IEEE Internet of Things Journal, 349-359.

CISCO. (2013). Education and the Internet of Everything. Cisco Consulting Services and Cisco EMEAR Education Team, 1-15.

Culjak, I., \& Abram, D. (2012). A brief introduction to OpenCV. MIPRO, 1725-1730.

D. Guinard, V. T. (2010). Interacting with the soa-based internet of things: Discovery, query, selection, and on-demand provisioning of web services. Services Computing, IEEE, 223 235 .

Department of Electrical and Electronic Engineering - University of Cagliari. (2015). The Virtual Object the Internet of Things. IEEE Communications Surveys \& Tutorials, 1-12.

García, M. E. (2009). Diseño e implementación de una herramienta de detección facial. México.

I. F. Akyildiz, J. X. (2007). "A survey of mobility management in next-generation all-ip-based wireless systems. Wireless Communications, IEEE,, 16 -28.

ITU. (2005). ITU.ORG. Obtenido de http://www.itu.int/osg/spu/publications/internetofthings/ 
ITU. (2012). ITU. Obtenido de http://www.itu.int/osg/spu/publications/internetofthings/

Jaffey, T. (febrero de 2014). Eclipse, Retrieved from MQTT and CoAP, IoT Protocols. Obtenido de http://eclipse.org/community/eclipse_newsletter/2014/february/article2.php

Matuska, S., Hudec, R., \& Miroslav, B. (2012). The Comparison of CPU Time Consumption for Image Procession Algorithm in Matlab and OpenCV. IEEE, 75 - 78.

Michele Nitti, V. P. (2015). The Virtual Object as a Major Element of the Internet of Things: a Survey. IEEE, 1-12.

Paci , F., Brunelli, D., \& Benini, L. (2014). 0, 1, 2, many - A classroom occupancy monitoring system for smart public buildings. Design and Architectures for Signal and Image Processing (DASIP), 2014 Conference on, (págs. 1-6). Madrid.

Posada, M. G. (2015). Diseño de Prototipo de Recogida Automatizada de Bolos Mediante Brazo Robótico y Visión Artificial. Oviedo.

Shah, M. (1997). Fundamentals of Computer Vision. Orlando: University of Central Florida.

Sobrado, E. (2009). Sistema de visión artificial para el reconocimiento y manipulación de objetos utilizando un brazo robótico. Lima: Pontificia Universidad Católica de Perú.

Somayya Madakam, R. R. (Enero de 2015). Internet of Things (IoT): A Literature. Obtenido de http://file.scirp.org/pdf/JCC_2015052516013923.pdf

Vélez, J., Moreno, A., Sánchez, A., \& Sánchez, J. (2003). Visión por Computador. Universidad Rey Juan Carlos.

Zanella, A., Bui, N., Castellani, A., Vangelista, L., \& Zorzi, M. (2014). Internet of Things for Smart Cities. IEEE INTERNET OF THINGS JOURNAL, 22-32. 